\title{
Granulomatous lesions of the skin
}

\author{
Gautam K ${ }^{1}$, Pai RR ${ }^{2}$, Bhat $\mathrm{S}^{2}$ \\ ${ }^{1}$ Department of Pathology, Nepal Medical College, Kathmandu,Nepal \\ ${ }^{2}$ Department of Pathology,Kasturba Medical College, Mangalore, India
}

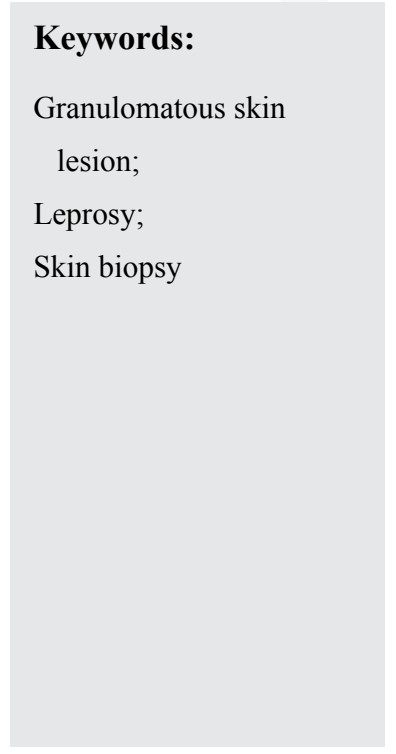

\begin{abstract}
Background: Granulomatous skin lesions often present as a diagnostic challenge to dermatopathologists due to various modes of presentation and identical histological picture produced by several causes. The aim of the study was to study different granulomatous skin lesions and to determine the relative frequency, the level of clinicopathologic concordance and to compare our results with those of other workers.
\end{abstract}

Materials and Methods: A retrospective analysis of skin biopsies received over a period of two years from January 2007 to December 2008; was performed, and cases of granulomatous dermatitis reported on histopathological examination were reviewed along with special stains.

Results: Out of a total of 1590 skin biopsies 106 (6.67\%) cases were found to have a granulomatous reaction. It was common in males $(63.21 \%)$ with most occurring in the fourth to fifth decades. Majority of cases (79 cases, $74.5 \%)$ were categorized as infectious granulomatous lesions with predominance of leprosy (63 cases, 79.7\%) followed by tuberculosis (6 cases, 7.6\%). An overall clinicopathologic concordance was seen in $97 \%$ of cases of leprosy.

Conclusion: In this study leprosy is the most common cause of granulomatous skin lesions. It can be concluded that histopathology plays an important role in classification of leprosy, and in diagnosis and management of a variety of granulomatous skin diseases. Special stains play a supportive role in infectious granulomas.

\section{INTRODUCTION}

The granulomatous reaction pattern is defined as a distinctive inflammatory pattern characterized by the presence of granulomas. Granulomas are relatively discrete collections of histiocytes or epithelioid histiocytes with variable numbers of admixed multinucleated giant cells and inflammatory cells. ${ }^{1}$ According to Dorland, the term "granulomatous" was expressed initially by Virchow to

\author{
Correspondence: \\ Dr. Keyoor Gautam, MD \\ Department of Pathology, Nepal Medical College, \\ Kathmandu, Nepal \\ Email: keyoor21@hotmail.com
}

describe a tumor-like mass or nodule of granulation tissue. ${ }^{2}$

Granulomatous skin lesions often present as a diagnostic challenge to dermatopathologists due to various modes of presentation and identical histological picture. The occurrence of different types of granulomatous lesions of the skin varies according to the geographical location. This is a comparative study of different granulomatous lesions of the skin in our geographical location.

The aim of this study was to determine the relative frequency of granulomatous lesions of the skin, the level of clinicopathologic concordance. 


\section{MATERIALS AND METHODS}

This was a retrospective analysis of 106 skin biopsies received in the Department of Pathology, Kasturba Medical College, Mangalore over a period of 2 years from January 2007 to December 2008. Clinical information and clinical diagnosis were noted. Sections stained with hematoxylin and eosin along with other necessary special stains were studied. The granulomas were subclassified into sarcoidal, tuberculoid, necrobiotic, suppurative, foreign body and miscellaneous granulomas. ${ }^{1}$

\section{RESULTS}

Out of 1590 skin biopsies, granulomatous lesions of the skin constituted 106 cases $(6.67 \%)$. Out of them, 67 (63.21\%) were males and $39(36.79 \%)$ were females with a male to female ration of 1.7:1. Ages ranged from 11 to 84 years with a mean age of 47.5 years. Maximum number of cases occurred in the fourth decade followed by fifth decade. Majority of the cases $(74.5 \%)$ were categorized as infectious granulomatous lesions, where as the rest (25.5\%) as non-infectious granulomatous lesions.

Out of a total of 106 cases, 73 (68.9\%) showed tuberculoid granulomas, $20(18.9 \%)$ had foreign body granulomas, $4(3.7 \%)$ each had necrobiotic and miscellaneous/nonspecific granulomas, $3(2.8 \%)$ had suppurative granulomas and $2(1.9 \%)$ had sarcoidal granulomas. Leprosy (63 cases, $59.43 \%$ ) constituted majority of the cases of granulomatous skin lesions.

The most common site of granuloma was the upper extremity (23 cases, $21.7 \%$ ). Most of the lesions of tuberculoid leprosy were in the upper extremity, while histiocytic leprosy showed whole body involvement. The overall concordance between clinical and histopathological diagnosis of different types of granulomatous lesions of the skin was $88.7 \%$.

In the present study of 63 leprosy cases, Borderline Tuberculoid (30 cases, 47.6\%) (fig.1A\&B) predominated followed by Indeterminate leprosy ( 8 cases, 12.7\%). The least common were histioid leprosy (fig 2) and Hansen's disease without clinical sub-classification (1 case each, $1.6 \%)$.

The overall level of concordance between the clinical and histopathological diagnosis was $96.8 \%$. However, after excluding the 32 cases mentioned above, the overall concordance was $61.3 \%$. Six cases of cutaneous tuberculosis were recorded and were typified as Lupus Vulgaris (LV, 2 cases), tuberculosis cutis orificialis (TCO, 2 cases), miliary tuberculosis (1 case) and tuberculosis verrucosa cutis (TVC, 1 case). Stain for acid fast bacilli (AFB) was positive in the case of TCO.

Two cases of cutaneous sarcoidosis were recorded in this study. Both were females aged 49 and 51 years old. Initial diagnosis of sarcoidosis was made on biopsy and further substantiated by radiological findings.

Cutaneous leishmaniasis comprised $3.7 \%$ of the cases studied. All four cases were seen in males with age range of 22 to 75 years. Upper extremities were predominantly involved with pain and itchiness as the major complaints. Biopsy showed presence of Leishnania Donavani (LD) bodies on routine HE stain (fig.3). However, in one case Giemsa stain was equivocal.

Cutaneous chromoblastomycosis comprised $2.8 \%$ of the cases studied. All three cases were seen in males with age ranging from 52 to 74 years. Majority of the lesions involved in the lower extremity. Dark brown "copper penny" bodies were characteristic finding in all cases (fig. 4A\&B).

Granuloma annulare comprised four (3.7\%) of the 106 cases studied. Three of the four patients were females with predominance in the younger age group. Majority of the cases presented with localized lesions.

Foreign body granuloma comprised $18.9 \%$ of the total cases. Majority of the cases were of epidermal cyst with wide age distribution occurring predominantly on the head and neck area, and the trunk.

\section{DISCUSSION}

The studies focusing on the different kinds of granulomatous lesions of the skin were conducted by various authors. ${ }^{3-5}$

The present study showed agreement to Dhar's study with respect to the male predominance of granulomatous lesions of the skin. ${ }^{6}$ Infectious granulomatous lesions were predominant in the present study in accordance to the study done by Bal et al. ${ }^{3,4}$ In Zafar's study, ${ }^{5}$ the most common site of granulomatous skin disease $(23.57 \%)$ was in the head and neck region. Whereas in our study majority occurred in the upper extremity $(22.43 \%)$.

Tuberculoid granuloma was the most common in all four studies (Table 1). Though the occurrence of tuberculoid granuloma was lower in this study, it still was the most common type of granuloma present

Out of a total of 515 cases of infectious granuloma in Bal's study, $373(72.4 \%)$ were leprosy cases followed by 119 (23.1\%) cases of cutaneous tuberculosis. ${ }^{3}$ In our study 63 $(79.7 \%)$ cases were leprosy followed by $6(7.6 \%)$ cases of cutaneous tuberculosis.

A major bulk of the cases comprised of leprosy which were typed and analyzed for clinical and histopathological concordance. Skin lesions in leprosy were more common in males. There were $41(65.08 \%)$ males and 22 ( $34.92 \%)$ 


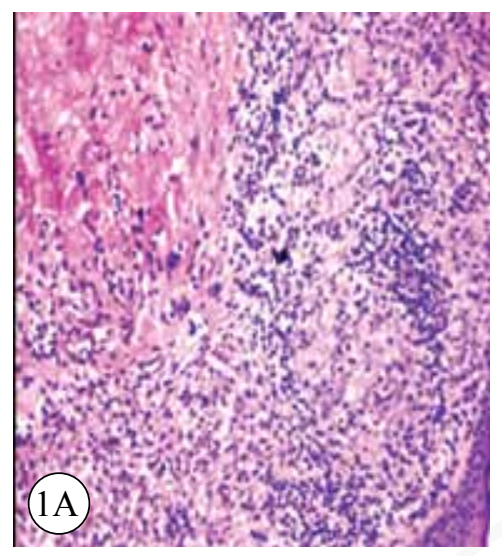

Figure 1A: Borderline tuberculoid leprosy. Epithelioid cell granulomas with moderate number of lymphocytes (HE stain, X100).

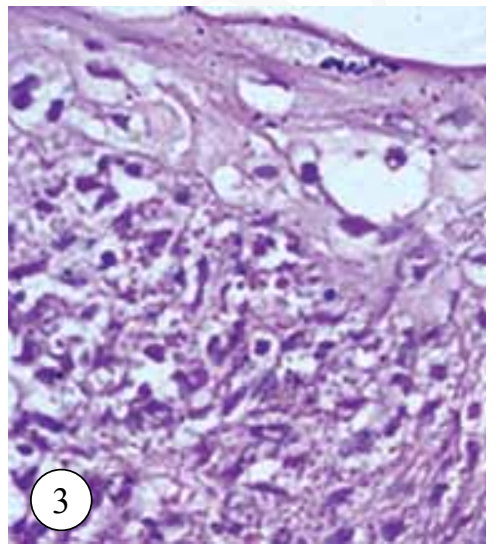

Figure 3: Leishmaniasis. Dense, diffuse infiltrate of histiocytes and lymphocytes with LD bodies (HE stain, X400).

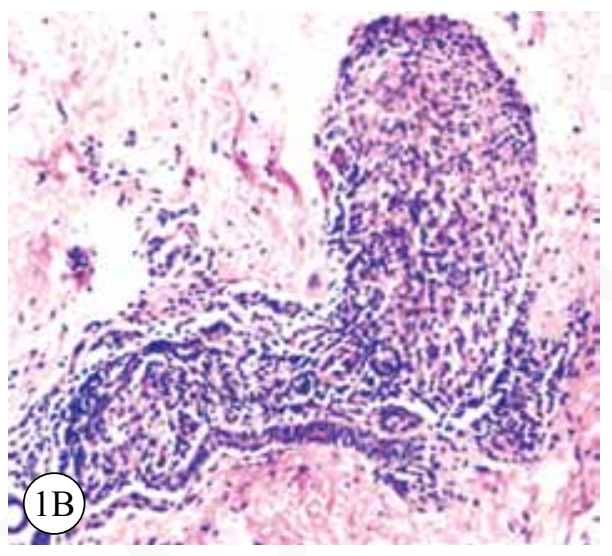

Figure 1B: Granulomas involving the nerves (HE stain, X100)

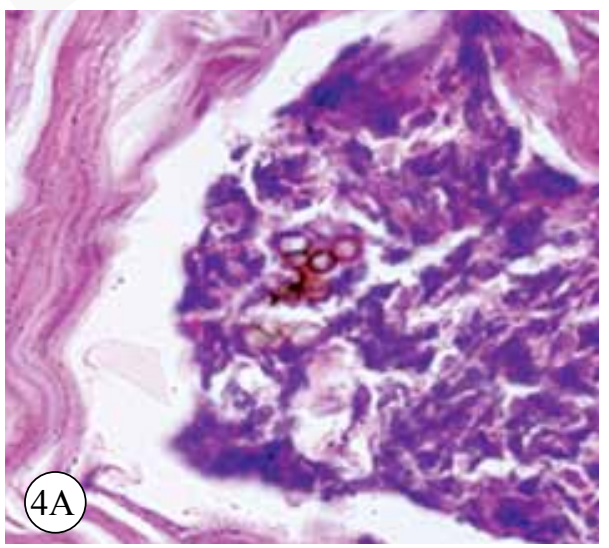

Figure 4A: Chromoblastomycosis.Pigmented spores resembling "copper pennies" (HE stain, X400).

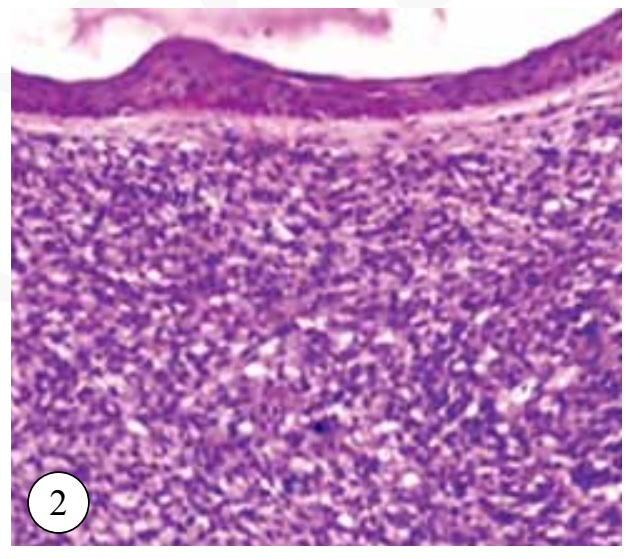

Figure 2: Histioid leprosy. Thinned out epidermis with an expansile lesions in the dermis (HE stain, X50).

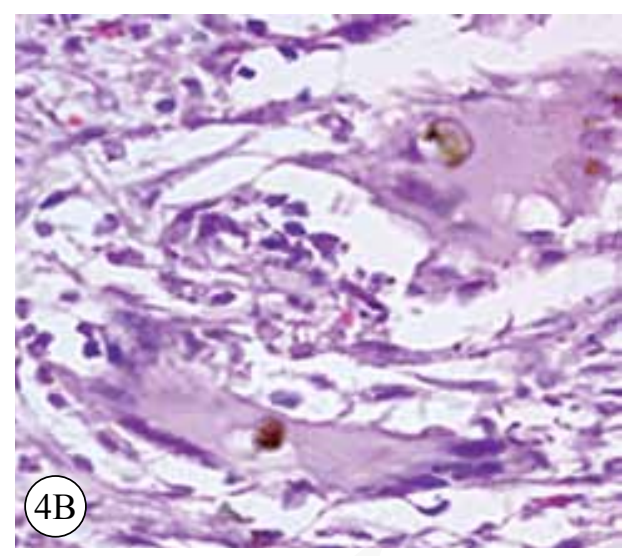

Figure 4B: Pigmented spores seen within multinucleated giant cells (HE stain, X400). females with a male to female ratio of $1.8: 1$. These results were comparable with Jayalakshmi who also found male preponderance $(73.68 \%)$ with a male to female ratio of 2.8:1. ${ }^{7}$ Nadkarani and Rege ${ }^{8}$, Moorthy et $\mathrm{al}^{9}$ also found males to be involved more by leprosy of the skin. Borderline tuberculoid leprosy was the most common lesion encountered in our study in accordance to various other studies. ${ }^{3,7-9}$

In most of the studies referred to, the correlation was better at the lepromatous pole than the tuberculoid pole (Table 2). The present study showed an exact opposite finding with a higher correlation at the tuberculoid pole.

Bhatia et $\mathrm{al}^{10}$ observed that upon combining some of the groups, the concordance for TT/BT group was $80 \%$ and for the BL/LL group it was $93 \%$. The researchers have stated that since both TT and BT are considered paucibacillary and $\mathrm{LL}$ or $\mathrm{BL}$ are considered multibacillary for treatment purpose, differentiating TT from BT or BL from LL was perhaps therapeutically irrelevant. ${ }^{10}$ Upon combining some of the groups in the present study, the concordance for TT/ BT group was $52.6 \%$ and for the BL/LL group it was $25 \%$, which was significantly lower than the study. ${ }^{10}$

Differentiating Non-specific dermatitis from indeterminate leprosy poses as a common problem to dermatopathologists. The following can serve as a guideline:

1. Irregular (pseudo-epitheliomatous) hyperplasia of epidermis

2. Neutrophils among the infiltrate

These two almost exclude a diagnosis of early leprosy. The following features are typical of non-specific dermatitis and make early leprosy rather unlikely:

1. Cellular infiltrate (not granuloma) with a predominantly perivascular distribution and very little around skin adnexal structures 
2. Much edema either around blood vessels or in the epidermis or both

3. Much melanin pigment in melanophores in the superficial dermis when the basal layer of epidermis is intact. $^{11}$

Two out of 106 cases were diagnosed as cutaneous sarcoidosis in the present study. Sarcoidosis was rare in the series by Zafar and common in other studies. ${ }^{4-6}$ Both patients were female and the initial diagnosis of sarcoidosis was made in skin biopsy and further substantiated by radiological evidence of pulmonary involvement and mediastinal lymphadenopathy. Non-caseating granulomas were seen in the dermis in both the cases with similarity to other series with dermal predominance. ${ }^{6}, 12$ A negative Montoux test in one of the cases helped to rule out a differential diagnosis of cutaneous tuberculosis. Cutaneous anergy is a feature in sarcoidosis. An interesting observation in one of the cases was perineural invasion by

the granulomas suggesting that it is not only leprosy that has perineural involvement. Reticulin stain can also be done to show the increase in reticulin fibers surrounding and permeating the epithelioid granulomas. In addition, Masson's Trichrome staining can be used to demonstrate fibrinoid necrosis. ${ }^{13}$ Chest X-ray and serum calcium levels are required for confirmation of the diagnosis. ${ }^{4}$

Foreign material may be a frequent nidus of cutaneous granuloma formation. ${ }^{12,14}$ Both the cases were studied under polarized microscope to rule out presence of foreign material. Atypical histological findings and foreign bodies are more common than initially expected in cutaneous sarcoidosis. ${ }^{12}$ The ultimate diagnosis will require clinical correlation, laboratory investigations, chest $\mathrm{x}$-ray as well as available tissue culture. ${ }^{5,13,14}$

In the present study 6 out of 1590 cases of skin biopsies were diagnosed as tuberculosis $(0.38 \%)$. The worldwide incidence of tuberculosis varies from 0.1 to $1 \%$ of all cutaneous conditions. In Pakistan the frequency of cutaneous tuberculosis is high (3.7\%).6 A female preponderance was observed (4/6) in our series similar to other studies. ${ }^{6,15,16}$

Among the six cases in the present study the lesions were typified as LV ( 2 cases), TCO ( 2 cases), cutaneous miliary tuberculosis (1 case) and TVC (1 case).

LV was the commonest form in some studies, ${ }^{4,6,17}$ whereas scrofuloderma predominated in others, ${ }^{18}$ particularly in childhood. ${ }^{19} \mathrm{LV}$ affects primarily the head and neck region, although in Southeast Asia it appears to be more common on the extremities and buttocks. ${ }^{1,17}$

TCO constituted $7 / 47$ cases typified in the study by Zafar et al. ${ }^{5}$ We encountered two cases, one with painful vulval, inguinal and perianal lesions and the other with multiple perianal eczematous lesions. In the former case, histopathologically a dense mixed inflammatory infiltrate was seen with ill formed granulomas. Stain for AFB was negative. This patient also had biopsy proven granulomatous cervicitis. Treatment with ATT showed only partial response. The patient refused colonoscopic evaluation to rule out the possibility of Crohn disease. In the latter case, there were distinctive tuberculous granulomas with AFB positivity.

Cutaneous manifestations of miliary tuberculosis are rare. It presents with erythematous papulo pustular skin eruption and is described in patients with HIV infections. ${ }^{20,21}$ A 51 year old lady presented with papulo pustular lesion with

Table1: Comparison of the types of granuloma in different studies

\begin{tabular}{lccccccc}
\hline Studies & Tuberculoid & Sarcoidal & Foreign body & Suppurative & Necrobiotic & Miscella-neous \\
\hline Dhar and Dhar (2002) & $77.3 \%$ & $13.7 \%$ & - & $9 \%$ & - & - \\
\hline Bal et al. (2006) & $87.7 \%$ & $2.6 \%$ & $1.7 \%$ & $2.9 \%$ & $2.7 \%$ & $2.4 \%$ \\
\hline Zafar et al. (2008) & $92.7 \%$ & $1.6 \%$ & $3.3 \%$ & $1.6 \%$ & $0.8 \%$ & - \\
\hline Present study & $68.9 \%$ & $1.9 \%$ & $18.9 \%$ & $2.8 \%$ & $3.7 \%$ & $3.7 \%$ \\
\hline
\end{tabular}

Table2: Comparative study of clinico-pathological correlation of different studies

\begin{tabular}{|c|c|c|c|c|c|c|}
\hline Studies & In* & TT $\dagger$ & BT & BB§ & BL $\|$ & LLף \\
\hline Bhatia et al. (1993) & $36 \%$ & $50 \%$ & $77 \%$ & $26 \%$ & $43 \%$ & $91 \%$ \\
\hline Nadkarani and Reges (1999) & $93 \%$ & $88 \%$ & $80.7 \%$ & $69.9 \%$ & $81.3 \%$ & $95.3 \%$ \\
\hline Kalla et al. (2000) & - & $75.6 \%$ & $44.2 \%$ & $37 \%$ & $43.7 \%$ & $76.7 \%$ \\
\hline Moorthy et al. (2001) & $20 \%$ & $46.15 \%$ & $66.54 \%$ & $50 \%$ & $70 \%$ & $80 \%$ \\
\hline Sharma et al. (2008) & $100 \%$ & $47.37 \%$ & $53.01 \%$ & $37.35 \%$ & $58.82 \%$ & $75.86 \%$ \\
\hline Present study & $100 \%$ & $33 \%$ & $72.2 \%$ & $100 \%$ & - & $50 \%$ \\
\hline
\end{tabular}

*Indeterminate leprosy $\uparrow$ Tuberculoid leprosy $\ddagger$ Borderline Tuberculoid leprosy $\S$ Mid Borderline leprosy $\|$ Borderline Lepromatous leprosy $\uparrow$ Lepromatous leprosy 
discharging sinuses all over the body. Histopathology showed caseation granulomas with superficial dermal abscess. Stain for AFB was negative, however no history was available regarding tuberculosis of the lung or any other organ.

TVC is a common clinical pattern. ${ }^{5}$ An eighteen year old boy presented with verrucous papule on dorsum of the hand which on histopathology showed characteristic epidermal changes and mixed inflammatory granuloma in the deep dermis. Sporotrichosis was considered in differential diagnosis, however both AFB and PAS stain for fungus were negative. Culture and PCR play an important role in correctly diagnosing and treating such cases. ${ }^{21}$

Ziehl Neelsen stain for AFB is indicated in all suspected cases of cutaneous tuberculosis. AFB were seen in $25.3 \%$ of caseating and $2.8 \%$ of non-caseating granulomas. ${ }^{3}$ Tubercle bacilli are very rarely demonstrated in cases of LV as well as TVC. ${ }^{6}$ In cases of scrofuloderma, they may be found occasionally. They are known to be positive in TCO. ${ }^{1,6}$ Among the six cases in our study, only one case of TCO (16.6\%) was AFB positive.

Cutaneous leishmaniasis comprised $3.7 \%$ of the cases studied which was midway in comparison to the studies done by Zafar (7.3\%) and Bal (1.16\%). ${ }^{3,5}$ All four cases were seen in males and predominantly on the upper extremity. Itchiness and pain were the common symptoms. On histopathological examination LD bodies were identified on routine HE sections but had equivocal results in one case with Giemsa and PAS stains. Hence a diagnosis suggestive of cutaneous leishmaniasis was given. In the series published by Bal et al, LD bodies were identified in only $50 \%$ of cases. ${ }^{3}$ Leishmanin skin test (positive in $80 \%$ cases) and exudates staining with Giemsa or Wrights stain can be performed as an ancillary test. The definitive diagnosis rests on the isolation of organisms by smears and culture or its identification in tissue sections. ${ }^{22}$ In the study by Bal et $\mathrm{al}^{3}$ they were able to identify LD boides in only $50 \%$ of the cases. It is usually difficult to detect LD bodies in paraffin sections, but plasma cell histiocytic infiltrate can suggest the diagnosis. ${ }^{3}$

Granuloma annulare comprised four (3.7\%) of the 106 cases studied which was similar in incidence with other case series. ${ }^{4,5}$ Majority of the patients were young with a female predominance similar to the study done by Friedman-Birnbaun, however in the study done by Mohan et al. a male predominance was found. ${ }^{4,23}$ Clinically three fourths of the cases were of localized granuloma annulare. On histopathological examination three of the four cases showed dominance of an interstitial pattern. Palisading pattern of presentation was seen in $25 \%$ of the total cases, similar to other case series. ${ }^{23,24}$ In cases where mucin is clearly not apparent, a colloidal iron and alcian blue stain can be done.
Cutaneous chromoblastomycosis is a chronic localized infection of the skin and subcutaneous tissue with a recrudescent character, potentially associated with the growth of epidermoid carcinoma in affected regions and a poor quality of life and work incapacity to the patient. ${ }^{25}$ Farmers are usually affected with predilections for old age and male predominance. The lesions commonly involve the lower extremities. ${ }^{25-27}$ Two out of three cases encountered in our study were farmers and all were elderly male with involvement of the lower extremities.

All three cases showed pseudoepitheliomatous hyperplasia, dermal microabscess, epithelioid cells and multinucleated giant cells along with dark brown "copper penny" bodies (fig.4). A positive $\mathrm{KOH}$ mount may also aid in the identification of the fungus. In cases where fungal organisms are not identified but a high index of suspicion is present, a fungal culture is always the confirmatory test. ${ }^{26}$

Foreign body granuloma comprised $18.9 \%$ of the total cases studies, which was significantly higher compared to other case series. ${ }^{4,5}$ Majority were epidermal cyst with a clinicohistopathologic concordance of $94.4 \%$. Majority of the lesions were seen in the head and neck area, and the trunk. When an epidermal cyst ruptures and the contents of the cyst are released into the dermis, a considerable foreign-body reaction with numerous multinucleated giant cells results, forming a keratin granuloma. The foreign body reaction usually causes disintegration of the cyst wall and may lead to a pseudocarcinomatous proliferation in remnants of cyst walls.

\section{CONCLUSION}

Infections form an important cause of granulomatous skin lesions with leprosy as the leading etiology. The clinicohistopathologic concordance of granulomatous skin lesions is high (88.7\%) with a clinicopathologic concordance of $61.3 \%$ for leprosy in this study. It can be concluded that histopathology plays an important role in sub-classification and management of the disease.

Granulomatous skin lesions have various modes of presentation. A classical clinical picture may not always be present. Skin biopsies definitely help in arriving at a diagnosis, provided that a proper history and clinical correlation is available. Special stains play a supporting role in infectious granulomas. Culture and PCR are indicated in certain cases.

\section{REFERENCES}

1. Weedon D. The granulomatous reaction pattern. In: Weedon D (ed). Skin Pathology, 2nd ed. Philadelphia: Churchill Livingstone; 2002. pp193-220.

2. Hirsh BC and Johnson WC. Concepts of granulomatous inflammation. Int J Dermatol 1984; 23: 90-9. 
3. Bal A, Mohan H, Dhami GP. Infectious granulomatous dermatitis: a clinicopathological study. Indian J Dermatol 2006;51:217-20.

4. Mohan H, Bal A, Dhami GP. Non-infectious granulomatous dermatitis: a clinicopathological study. J Cutan Pathol 2006; 33:76771.

5. Zafar MNU, Sadiq S, Menon MA. Morphological study of different granulomatous lesions of the skin. J Pak Assoc Dermatol 2008;18:21-8.

6. Dhar S, Dhar S. Histopathological features of granulomatous skin diseases: an analysis of 22 skin biopsies. Indian J Dermatol 2002;47:88-90.

7. Pailoor J. Histopathology of skin lesion in leprosy. Malaysian J Pathol 1980; 3: 39- 45.

8. Nadkarni NS Rege VL. Significance of histopathological classification in leprosy. Indian J Lepr 1999;71:325-31.

9. Moorthy BN, Kumar P, Chatura KR, Chandrashekhar HR, Basavaraja PK. Histopathological correlation of skin biopsies in leprosy. Indian J Dermatol Venereol Leprol 2001;67:299-301.

10. Bhatia AS, Katoch K, Narayanan RB, Ramu G, Mukherjee A, Lavania RK. Clinical and histopathological correlation in the classification of leprosy. Int J Lepr 1993;61:433-8.

11. Ridley DS. Skin Biopsy in Leprosy. 3rd ed London: Documenta Geigy; 1976.pp37-58.

12. Mangas C, Fernandez-Figueras MT, Fite E, Fernandez-Chico $N$, Sabat $M$ and Ferrandiz C. Clinical spectrum and histological analysis of 32 cases of specific cutaneous sarcoidosis. J Cutan Pathol 2006;33:772-7.

13. Manonukul J, Wanitphakdeedecha R, Wisuthsarewong W, Thirapote P. Histopathologic aid to diagnosis of sarcoidosis: report of 8 cases. J Med Assoc Thai 2006;89:864-71.

14. Ball NJ, Kho G T, Martinka M. The histological spectrum of cutaneous sarcoidosis: a study of twenty eight cases. J Cutan Pathol 2003;31:160-8
15. Khan Y, Anwar J, Iqbal P, Kumar A. Cutaneous tuberculosis: a studies of ten cases. J Pak Assoc Dermatol 2001;11:6-10.

16. Yasmeen H, Kanjee A. Cutaneous tuberculosis: a three year prospective study. J Pak Med Assoc 2005;55:10-3.

17. Kumar B, Muralidhar S. Cutaneous tuberculosis: a twenty-year prospective study. Int J Tuberc Lung Dis 1999;3:494-500.

18. Farina MC, Gegundez MI, Pique E et al. Cutaneous Tuberculosis: A clinical, histopathologic and bacteriologic study. J Am Acad Dermatol 1995;33:433-40.

19. Kumar B, Rai R, Kaur I, Sahoo B, Muralidhar S, Radotra BD. Childhood cutaneous tuberculosis: a study over 25 years form northern India. Int J Dermatol 2001;40:26-32.

20. High VA, Evans CC, Hoang MP. Cutaneous miliary tuberculosis in two patients with HIV infection. J Am Acad Dermatol 2004;50:S110-3.

21. Antinori S, Galimberti L, Tadini GL et al. Tuberculosis cutis miliaris disseminate due to multidrug-resistant mycobacterium tuberculosis in AIDS patients. Eur J Clin Microbiol Infect Dis 1995;14:911-4.

22. Farah FS, Malak JA. Cutaneous Leishmaniasis. Arch Derm 1971;103:467-73.

23. Friedman-Birnbaum R. Generalized and localized granuloma annulare. Int J Dermatol 1986;25:364-6.

24. Dabski K, Winkelmann R K. Generalized granuloma annulare: Histopathology and immunopathology: systematic review of 100 cases and comparison with localized granuloma annulare. J am Acad Dermatol 1989;20:28-39.

25. Minotto R, Bernardi CS, Mallmann LF, Edelweiss MI, Scroferneker ML. Chromoblastomycosis: a review of 100 cases in the state of Rio Grande do Sul, Brazil. J Am Acad Dermatol 2001;44:585-92.

26. Pradhan SV, Talwar OP, Ghosh A, Swami RM, KC SR, Gupta S. Chromoblastomycosis in Nepal: A study of 13 cases. Indian J Dermatol Venereol Leprol 2007; 73: 176-8.

27. Sharma NL, Sharma RC, Grover PS, Gupta ML, Sharma AK, Mahajan VK. Chromoblastomycosis in India. Int J Dermatol 1999;38:846-51. 